PROCEEDINGS OF THE

AMERICAN MATHEMATICAL SOCIETY

Volume 132, Number 6, Pages 1845-1848

S 0002-9939(03)07359-3

Article electronically published on December 23, 2003

\title{
A CHARACTERIZATION OF DISCRETE GROUPS
}

\author{
GIOVANNI RANIERI \\ (Communicated by N. Tomczak-Jaegermann)
}

\begin{abstract}
The purpose of this article is to prove the following result. Let $G$ be a locally compact group, $\mathcal{A}(G)$ the Fourier algebra of $G$, and $\mathcal{S}(G)=\{u \in$ $\mathcal{A}(G): \exists c>0$ such that $\left.\|u v\|_{\mathcal{A}(G)} \leq c\|v\|_{\infty} \quad \forall v \in \mathcal{A}(G)\right\}$. Then $G$ is a discrete group $\Longleftrightarrow \mathcal{S}(G) \neq\{0\}$.
\end{abstract}

\section{INTRODUCTION}

In 1957, S. Helgason proved in [4] that if $G$ is a separable, unimodular, locally compact, noncompact, connected group, then a spectrally continuous operator on $L^{1}(G)$ commuting with all right translations is identically zero. In 1964 , S. Sakai generalized this result to any noncompact, locally compact group (see [6]).

In this article, we consider the dual problem, namely the spectrally continuous operators of $\mathcal{A}(G)$ defined by elements of $\mathcal{B}(G)$. Let $G$ be a locally compact group, and denote by $\mathcal{S}(G)$ the set of all $u \in \mathcal{A}(G)$ that define a spectrally continuous multiplication of $\mathcal{A}(G)$. Then, if $G$ is a discrete group, $\mathcal{S}(G) \neq\{0\}$ because it contains $l^{2}(G)$. We show here that the converse is true.

This result may be embodied in a much more general result about commutative Banach algebras. Therefore the main result is Theorem 2.1, and the announced result is Corollary 3.1. The author is grateful to the referee for this improvement (see the Acknowledgments).

\section{SpeCtrally CONTINUOUS MUltipliers FOR A COMMUTATIVE BANACH ALGEBRA}

For a commutative Banach algebra $\mathcal{A}$, we denote by $\Delta(\mathcal{A})$ the structure space of $\mathcal{A}$, endowed with the usual topology. For $x \in \mathcal{A}, \hat{x}$ is the Gelfand transform of $x$, and $\hat{\mathcal{A}}$ is the range of the Gelfand homomorphism $\mathcal{A} \rightarrow \mathcal{C}_{0}(\Delta(\mathcal{A}))$.

Theorem 2.1. Let $\mathcal{A}$ be a regular semisimple commutative Banach algebra and let

$$
\mathcal{S}(\mathcal{A})=\left\{a \in \mathcal{A}:\|a x\| \leq c\|\hat{x}\|_{\infty} \text { for some } c>0 \text { and all } x \in \mathcal{A}\right\} .
$$

(i) If $\Delta(\mathcal{A})$ has an isolated point, then $\mathcal{S}(\mathcal{A}) \neq\{0\}$.

(ii) Suppose that $\mathcal{A}$ is an abstract $L$-space and that the closure of $\hat{A}$ in $\mathcal{C}_{0}(\Delta(\mathcal{A}))$ is closed under conjugation. If $\mathcal{S}(\mathcal{A}) \neq\{0\}$, then $\Delta(\mathcal{A})$ has isolated points.

Received by the editors October 9, 2002 and, in revised form, February 11, 2003.

2000 Mathematics Subject Classification. Primary 22D15.

(C)2003 American Mathematical Society 
Proof. To show $(i)$, let $\varphi$ be an isolated point of $\Delta(\mathcal{A})$. Since $\mathcal{A}$ is regular, there exists $a \in \mathcal{A}$ such that $\hat{a}(\varphi) \neq 0$ and $\hat{a}(\psi)=0$ for all $\psi \in \Delta(\mathcal{A}), \psi \neq \varphi$.

Then, for any $x \in \mathcal{A}, \varphi(a x-\varphi(x) a)=0$ and

$$
\psi(a x-\varphi(x) a)=\psi(a)(\psi(x)-\varphi(x))=0 \text { for each } \psi \neq \varphi .
$$

Since $\mathcal{A}$ is semisimple, it follows that $a x=\varphi(x) a$, and hence

$$
\|a x\|=|\varphi(x)|\|a\| \leq\|a\|\|\hat{x}\|_{\infty} \text { for all } x \in \mathcal{A} .
$$

Thus $a \in \mathcal{S}(\mathcal{A})$, and $(i)$ holds.

To establish $(i i)$, let $a$ be a nonzero element of $\mathcal{S}(\mathcal{A})$. Then $a$ defines a bounded linear map $T_{a}$ from $\left(\hat{\mathcal{A}},\|\|_{\infty}\right)$ into $\mathcal{A}$ by $T_{a} \hat{x}=a x$. Let $\overline{\mathcal{A}}$ be the closure of $\hat{A}$ in $\mathcal{C}_{0}(\Delta(\mathcal{A}))$. Of course, $T_{a}$ extends uniquely to a bounded linear map, also denoted $T_{a}$, from $\overline{\hat{\mathcal{A}}}$ into $\mathcal{A}$. Since by hypothesis $\overline{\hat{\mathcal{A}}}$ is a $\mathcal{C}^{*}$-algebra and $\mathcal{A}$ is an abstract $L$-space, $T_{a}$ is weakly compact by [6]. This implies that the map $L_{a}: x \mapsto a x$ from $\mathcal{A}$ into $\mathcal{A}$ is weakly compact. Indeed, denoting by $\mathcal{A}_{r}$ the closed ball of radius $r$ in a normed space $\mathcal{A}$,

$$
L_{a}\left(\mathcal{A}_{1}\right)=T_{a}\left(\hat{\mathcal{A}}_{1}\right) \subset T_{a}\left(\overline{\hat{\mathcal{A}}}_{1}\right),
$$

which is a relatively compact set in $\mathcal{A}$. Now, if $T$ is a bounded linear operator of an abstract $L$-space into itself that is weakly compact, then $T^{2}$ is strongly compact (see 1], p. 128, Corollary 1). So $L_{a}^{2}=L_{a^{2}}$ is strongly compact.

Let $0<\delta<\|a\|_{\infty}$ and $K=\left\{\varphi \in \Delta(\mathcal{A}):|\hat{a}(\varphi)|^{2} \geq \delta\right\}$, a compact subset of $\Delta(\mathcal{A})$. Let

$$
A_{K}=\{x \in \mathcal{A}: \operatorname{supp} \hat{x} \subseteq K\} .
$$

Since $\left|\widehat{a^{2}}\right| \geq \delta$ on $K$, there exists $b \in \mathcal{A}$ such that $\widehat{a^{2}}(\varphi) \hat{b}(\varphi)=1$ for all $\varphi \in K$ (see Theorem 3.6.15 of Rickart [5]). Then, for $x \in \mathcal{A}_{K}$,

$$
\hat{x}(\varphi)=\widehat{a^{2}}(\varphi) \hat{b}(\varphi) \hat{x}(\varphi) \text { for all } \varphi \in \Delta(\mathcal{A}),
$$

and hence $x=a^{2} b x$ since $\mathcal{A}$ is semisimple. Thus

$$
\left(\mathcal{A}_{K}\right)_{1}=a^{2} b\left(\mathcal{A}_{K}\right)_{1} \subseteq a^{2}\left(\mathcal{A}_{K}\right)_{\|b\|} \subseteq L_{a}^{2}\left(\mathcal{A}_{\|b\|}\right) .
$$

$L_{a}^{2}\left(\mathcal{A}_{\|b\|}\right)$ is a strongly compact subset of $\mathcal{A}$, and hence so is $\left(\mathcal{A}_{K}\right)_{1}$. It follows that $\mathcal{A}_{K}$ is finite dimensional.

It remains to show that the open subset $V=\left\{\varphi \in \Delta(\mathcal{A}):|\hat{a}(\varphi)|^{2}>\delta\right\}$ of $\Delta(\mathcal{A})$ is finite. Assuming the contrary, let $\varphi_{1}, \varphi_{2}, \ldots$ be an infinite sequence of pairwise distinct elements of $V$. Since $\mathcal{A}$ is regular, for each $n \in \mathbb{N}$ there exists $a_{n}$ of norm one in $\mathcal{A}$ such that $\widehat{a_{n}}\left(\varphi_{n}\right) \neq 0$ and $\widehat{a_{n}}$ is zero on $(\Delta(\mathcal{A}) \backslash V) \cup$ $\left\{\varphi_{1}, \ldots, \varphi_{n-1}\right\}$. Then $a_{n} \in \mathcal{A}_{K}$ for every $n \in \mathbb{N}$, and each finite sequence $a_{1}, \ldots, a_{n}$ is linearly independent. In fact, if $\sum_{j=1}^{n} \lambda_{j} a_{j}=0$, then

$$
0=\sum_{j=1}^{n} \lambda_{j} \widehat{a_{j}}\left(\varphi_{k}\right)=\sum_{j=1}^{k} \lambda_{j} \widehat{a_{j}}\left(\varphi_{k}\right) \text { for all } 1 \leq k \leq n,
$$

and since $\widehat{a_{k}}\left(\varphi_{k}\right) \neq 0$ it follows by induction on $k$ that $\lambda_{k}=0$ for all $k$. This contradicts the finite dimensionality of $\mathcal{A}_{K}$.

Corollary 2.2. Let $\mathcal{A}$ and $\mathcal{S}(\mathcal{A})$ be as in the theorem. 
(i) If $\Delta(\mathcal{A})$ is discrete, then $\mathcal{S}(\mathcal{A})$ contains all elements of $\mathcal{A}$ with finitely supported Gelfand transform. In particular, if $\Delta(\mathcal{A})$ is discrete and $\mathcal{A}$ is Tauberian, then $\mathcal{S}(\mathcal{A})$ is dense in $\mathcal{A}$.

(ii) Suppose that $\mathcal{A}$ is an abstract L-space and that $\overline{\hat{\mathcal{A}}} \subseteq \mathcal{C}_{0}(\Delta(\mathcal{A}))$ is closed under conjugation. If the hull of $\mathcal{S}(\mathcal{A})$ in $\Delta(\mathcal{A})$ is empty, then $\Delta(\mathcal{A})$ is discrete.

Proof. (i) is clear because $\mathcal{S}(\mathcal{A})$ contains the elements of $\mathcal{A}$ for which the Gelfand transform is the indicator function of an isolated point of $\Delta(\mathcal{A})$. Since $\Delta(\mathcal{A})$ is discrete, $\mathcal{S}(\mathcal{A})$ contains all elements of $\mathcal{A}$ with finitely supported Gelfand transform.

To establish (ii), let $\varphi \in \Delta(\mathcal{A})$. The hull of $\mathcal{S}(\mathcal{A})$ in $\Delta(\mathcal{A})$ is empty; so there exists an element $a \in \mathcal{S}(\mathcal{A})$ such that $\hat{a}(\varphi) \neq 0$. Let $\delta>0$ such that $\varphi \in V_{\delta}=$ $\left\{\psi \in \Delta(\mathcal{A})\right.$ such that $\left.|\hat{a}(\psi)|^{2}>\delta\right\}$.

But $V_{\delta}$ is an open finite set of $\Delta(\mathcal{A})$; so $\varphi$ is an isolated point of $\Delta(\mathcal{A})$.

\section{Characterization of Discrete groups}

Corollary 3.1. Let $G$ be a locally compact group and $\mathcal{A}(G)$ the Fourier algebra of G. Then

$$
G \text { is a discrete group if and only if } \mathcal{S}(G) \neq\{0\} .
$$

Proof. $\mathcal{A}(G)$ is a regular semisimple commutative Banach algebra whose structure space $\Delta(\mathcal{A}(G))$ can be identified with $G$ itself in such a way that $\hat{u}$ equals $u$ for all $u \in \mathcal{A}(G)$ (see [2]). Therefore $\mathcal{S}(\mathcal{A}(G))=\mathcal{S}(G)$.

We denote by $\mathcal{A}_{s a}(G)$ the real vector space of selfadjoint elements of $\mathcal{A}(G)$; if $u \in \mathcal{A}_{s a}(G)$, we consider the Jordan decomposition of $u, u=u^{+}-u^{-}$, where $u^{+}, u^{-} \in \mathcal{P}(G)$. Then $|u|=u^{+}+u^{-}$is the modulus of $u$. $\mathcal{A}_{s a}(G)$ with this modulus is a real Banach lattice that has the following property: if $u, v \in \mathcal{P}(G)$, then

$$
\|u+v\|=\|u\|+\|v\| .
$$

So $\mathcal{A}_{s a}(G)$ is a real Banach $L$-space, and $\mathcal{A}(G)=\mathcal{A}_{s a}(G)+i \mathcal{A}_{s a}(G)$ is the complexification of the real Banach lattice $\mathcal{A}_{s a}(G)$, so that $\mathcal{A}(G)$ is an abstract $L$-space. We refer to Schaefer ([7).

Moreover, $u \in \mathcal{A}(G)$ implies $\bar{u} \in \mathcal{A}(G)$. Thus, the statement of the corollary is an immediate consequence of the theorem.

If $G$ is a discrete group, we know that $\mathcal{S}(G)$ contains $l^{2}(G)$. Are $\mathcal{S}(G)$ and $l^{2}(G)$ equal for a discrete group? M. O. Gebuhrer and R. Szwarç recently gave an affirmative answer to this problem in a forthcoming publication (3]).

Remark 3.2. The existence of a nonzero multiplier defined by an element $u$ in $\mathcal{B}(G)$ is equivalent to the existence of such an operator defined by an element $u^{\prime}$ in $\mathcal{A}(G)$.

\section{ACKNOWLEDGMENTS}

The author wishes to thank the referee very gratefully: in fact, the first version of this article restricted its scope to the study of spectrally continuous operators of $\mathcal{A}(G)$. The referee enlarged the article considerably by pointing out what is now the main result of this paper, namely Theorem 2.1 . He also provided us with a sketch of the generalized proof as well as with numerous redactional improvements. 


\section{REFERENCES}

1. R. G. Bartle, N. Dunford, and J. Schwartz, Weak compactness and vector measures, Canadian J. Math. 7 (1955), 289-305. MR 16:1123C

2. P. Eymard, L'algèbre de Fourier d'un groupe localement compact, Bull. Soc. Math. France 92 (1964), 181-236. MR 37:4208

3. O. Gebuhrer and R. Szwarç, Alternating signs in orthogonal expansions, in preparation.

4. S. Helgason, Topologies of group algebras and a theorem of Littlewood, Trans. Amer. Math. Soc. 86 (1957), 269-283. MR 20:1930

5. C. Rickart, General theory of Banach algebras, The University Series in Higher Mathematics, D. van Nostrand Co., Princeton, N.J.-Toronto-London-New York, 1960. MR 22:5903

6. S. Sakai, Weakly compact operators on operator algebras, Pacific J. Math. 14 (1964), 659-664. MR 29:488

7. H. H. Schaefer, Banach lattices and positive operators, Die Grundlehren der mathematischen Wissenschaften, Band 215, Springer-Verlag, New York and Heidelberg, 1974. MR 54:11023

Institut de Recherche Mathématique Avancée, 7 rue René Descartes, 67000 StrasBOURG, FRANCE

E-mail address: GiovanniRanieri@aol.com 